\title{
Prominence Emission Lines Observed With SUMER and Two Ground-based Telescopes
}

\author{
C. R. de Boer \\ Max-Planck-Institut für Aeronomie, D-37191 Katlenburg-Lindau, \\ Germany \\ G. Stellmacher \\ Institut d'Astrophysique, 78bis Boulevard d'Arago, F-7501/4 Paris, France \\ E. Wiehr
}

Universitäts-Sternwarte, Geismarlandstr 11, D-37083 Göttingen, Germany

Abstract. Two sets of $\mathrm{H}, \mathrm{He}$, and $\mathrm{Ca}^{+}$emission lines were observed in a quiescent prominence simultaneously with the VTT and the Gregory telescope on Tenerife. At the same time, SUMER took two scans of low-ionized EUV emission lines.

The emission ratios of $\mathrm{Ca}^{+}$-to-Balmer lines from ground vary little in the prominence, indicating a largely constant gas-pressure. In contrast, the ratio of He-to-Balmer from the ground shows the (known) increase toward the prominence borders, indicating higher temperature there. Similarly, the two-dimensional distributions of the ratios S IV/N II and $\mathrm{C} \mathrm{III/He} \mathrm{I} \mathrm{show} \mathrm{pronounced} \mathrm{bright} \mathrm{prominence} \mathrm{rims.}$

The reduced He $537 \AA$ and He $584 \AA$ line widths are 2.6 and 3.6 times larger, respectively, than those of $\mathrm{He}_{3}$ and He $3888 \AA$. Explaining this by the optical thickness yields $\tau_{0}=10^{4}$ and $\tau_{0}=2 \cdot 10^{5}$ for the two EUV lines. The total He 584 emission amounts to $13 \mathrm{watt} / \mathrm{m}^{2}$ ster in the main prominence body where the $\mathrm{D}_{3}$ line yields 4 watt $/ \mathrm{m}^{2}$ ster; existing models, however, predict a factor 0.18 .

The widths of simultaneously observed optical lines with different atomic weights yield thermal and non-thermal broadening parameters of $T_{k i n} \approx 8000 \mathrm{~K}$ and $2.5<\xi<6.5 \mathrm{~km} / \mathrm{s}$. The EUV lines, however, show line widths which correspond to much higher temperatures and nonthermal velocities. Assuming for each ion the corresponding ionization temperature, the line widths require non-thermal velocities of $15-40 \mathrm{~km} / \mathrm{s}$ which is similar to values for the quiet corona.

\section{Introduction}

The transition between cool prominences and hot coronal material is not yet satisfactory explained. Cooling by Lyman radiation, ('cooling trap', Unsöld 1956), would 'rarefy' the surrounding corona. A smooth transition between cool 
prominence and hot corona, however, might cause a hot 'skin'. The known structuring of prominences offers the picture that either cool inner 'threads' are surrounded by hotter outer threads, or that each individual thread has a hot 'skin'. Such problems can be treated by EUV observations using the SUMER instrument along with ground based observations.

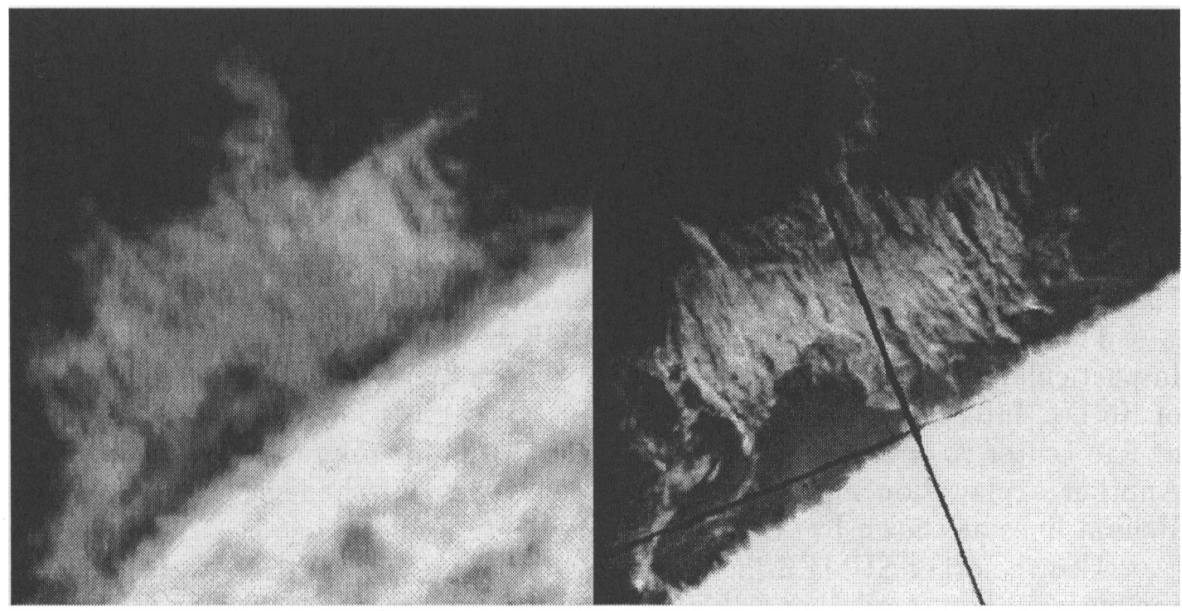

Figure 1. He I $584 \AA$ composite image from SUMER (left) and an $\mathrm{H}_{\alpha}$ image from the ground on June 28, 1996.

\section{Observations}

A quiescent hedgerow-type prominence at $\mathrm{E} 20^{\circ} \mathrm{S}$ was observed on June 28, 1996 with both German solar telescopes on Tenerife and with SUMER. The evacuated Gregory Coudé Telescope (GCT) was used to monitor the emission lines He $3888 \AA$ and $\mathrm{H}_{8} 3889 \AA$ simultaneously with $\mathrm{Ca}^{+} 8498 \AA$ on two $\mathrm{CCD}$ cameras integrating for $12 \mathrm{~s}$. With the Vacuum Tower Telescope (VTT), $\mathrm{H}_{\beta}, \mathrm{He}-\mathrm{D}_{3}$, and $\mathrm{Ca}^{+} 8542 \AA$ were simultaneously observed with three CCD cameras integrating for $3 \mathrm{~s}$. A fourth CCD took $\mathrm{H}_{\alpha}$ slit yaw images. The slit widths for both telescopes corresponded to $1.5 \mathrm{arcsec}$. The influence of wavelength-dependent refraction was avoided by orienting the slits in a direction perpendicular to the horizon. At the same time, SUMER was used to observe in a first scan the He I $584 \AA$ emission simultaneously with O I $1152 \AA$ C I $1158 \AA$, and C III $1175 \AA$. In a second series, SUMER took He I $537 \AA$ simultaneously with N II $1085 \AA$, S III $1077 \AA$ and S IV $1062 \AA$. Figure 1 shows a He I $584 \AA$ composite image from SUMER together with an $\mathrm{H}_{\alpha}$ slit jaw image from the VTT.

All CCD images were corrected for dark current and the gain matrices. For the ground-based spectra, the straylight aureole spectrum was subtracted. Finally, all prominence emissions were calibrated in units of the disc center intensities (Labs and Neckel 1970). The obtained emission lines were fitted by Gaussian profiles, from which shifts, widths, central intensities and integrated total emissions were determined. 
Scatter plot diagrams of the shifts of simultaneously observed lines from SUMER were used to verify that the lines originate from the same prominence regions in agreement with Wilk et al. (1993). Highest velocities up to $30 \mathrm{~km} / \mathrm{s}$ mainly occur in peripheral prominence regions. This is also found in the ground based spectra.

\section{Line Widths}

The widths of the emission lines from the ground based observations were used to separate thermal and non-thermal line broadening parameters. The ratio of the reduced 1/e-widths of $\mathrm{H}_{\beta} / \mathrm{Ca}^{+} 8542$ indicates a narrow temperature regime, $7500<K<8000 \mathrm{~K}$, but varying non-thermal velocities, $2.5<\xi<6.5 \mathrm{~km} / \mathrm{s}$.

The line widths observed with SUMER indicate much larger broadening. In particular, He $584 \AA$ has reduced widths which are 3.5 times larger than those of $\mathrm{He} \mathrm{D}_{3}$. Interpreting this as a saturation effect gives for the optical thickness at line center $\tau_{0}=2 \cdot 10^{5}$ for He I 584 and $7 \cdot 10^{3}$ for the fainter He I 537 line. Another explanation may be a different excitation mechanism for the various Helium lines as shown by Andretta and Jones (1997).

Also the other SUMER lines are much more broadened than expected from the optical lines. Assuming as kinetic temperature the corresponding ionization temperature, one obtains non-thermal velocities of $15-40 \mathrm{~km} / \mathrm{s}$. The range of non-thermal velocities would be smaller if the $O$ I line is assumed 'hotter' than $18,000 \mathrm{~K}$ and the C III line 'cooler' than $70,000 \mathrm{~K}$, in agreement with Seely et al. (1997) for the quiet corona.

The line widths depend slightly on the total emission $E_{t o t}$, but the largest widths do not necessarily occur at the largest $E_{\text {tot }}$. Instead, we find significantly broad lines for faint emissions, in contrast to Wilk et al. (1993). This might arise from Doppler-shifted superposed faint emissions.
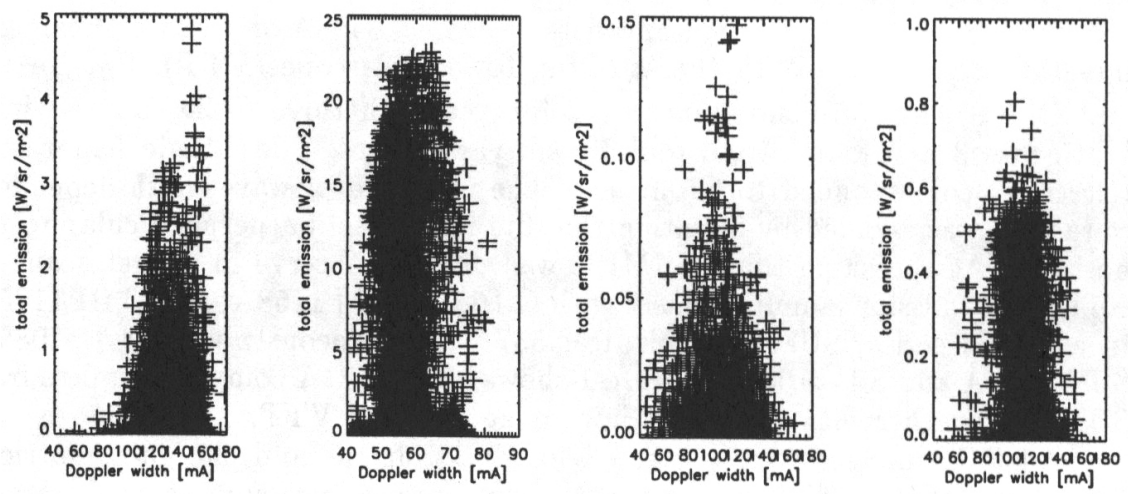

Figure 2. Dependence of the line width on the integrated emssion. From left: C III, He I 584, S IV, N II. 


\section{Integrated Line Emissions}

Among the integrated line emissions $E_{t o t}$, the ratio $\mathrm{Ca}^{+} / \mathrm{Balmer}$ is mostly sensitive to the gas-pressure. The almost constant ratio indicates small variations of the gas pressure in the prominence. The temperature-sensitive relation of the $\mathrm{He} \mathrm{D}_{3}$ versus the $\mathrm{H}_{\beta}$ emission (from the VTT spectra) shows the known branching (Stellmacher and Wiehr 1995) caused by an easier penetration of the ionizing and exciting UV radiation at peripheral prominence regions. In the spectra from the Gregory telecope, the two violet lines $\mathrm{He} 3888$ and $\mathrm{H}_{8}$ show a ratio of 1.6 which fit the relation by Stellmacher and Wiehr (1994) for $\mathrm{T}_{k i n} \approx 8000 \mathrm{~K}$. The mean ratio of the total emissions of He 3888 (from GCT) and He $\mathrm{D}_{3}$ (from VTT) is 0.06 , being nearly half of the calculated value 0.125 by Heasley et al. (1974). The $\mathrm{Ca}^{+} 8498$ line shows a mean prominence emission of 0.13 of that of $\mathrm{Ca}^{+}$ 8542, which is in good agreement with Landman and Illing (1977).

The SUMER lines show brightenings at the prominence periphery for the ratios of the C III/He I and S IV/N II emissions This brightening is only marginally

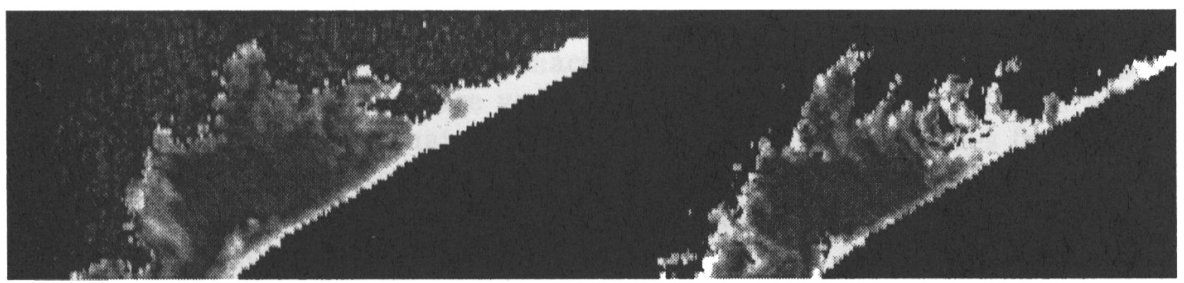

Figure 3. Total emission ratios of C III/He I 584 (left) and S IV/N II.

caused by broader lines but essentially due to an increased emission, similar to the larger $\mathrm{He} \mathrm{D}_{3} / \mathrm{H}_{\beta}$ ratio at the prominence borders.

For the total emissions of $\mathrm{He} I 584$ and $\mathrm{He} \mathrm{D}_{3}$ we find in the main body of the prominence mean values of 13 and $4 \cdot 10^{3}$ watt $/ \mathrm{m}^{2}$ ster. The ratio of 3.25 is 18 times higher than that calculated by Heasley et al. (1974).

Acknowledgments. Dr. P. Sütterlin kindly assited with the VTT observations and Drs. Wilhelm, Curdt and Marsch (MPAE) and Hammer (KIS) contributed helpful discussions. The Gregory and the VTT on Tenerife Island are operated by the Universitäts Sternwarte, Göttingen and, respectively the Kiepenheuer Institut für Sonnenphysik, Freiburg, at the Spanish Observatorio del Teide of the Instituto de Astrofisica de Canarias. SUMER is part of SOHO, the Solar and Heliospheric Observatory, of ESA and NASA. The SUMER project is financially supported by DARA, CNES, NASA, and the ESA PRODEX program.

\section{References}

Andretta V. and Jones H.P. 1997, ApJ, 489, 375

Heasley J.N., Mihalas D. and Poland A.I. 1974, ApJ, 192, 181

Labs D. and Neckel H. 1970, Sol. Phys. 17, 50 
Landman D.A. and Illing R.M.E. 1977, A\&A, 55, 103

Seely J.F., Feldman U., Schühle U., Wilhelm K. and Curdt W. 1997, ApJL 484, L87

Stellmacher G. and Wiehr E. 1994, A\&A, 286, 302

Stellmacher G. and Wiehr E. 1995, A\&A, 299, 921

Unsöld A. 1956, Physik der Sternwatmosphären, Springer/Heidelberg, p.700

Wilk J.E., Dere K.and Schmieder B. 1993, A\&A, 273, 267 